\title{
RESULTS FROM THE ETM COLLABORATION AT LIGHT DYNAMICAL QUARK MASSES
}

\author{
K. JANSEN \\ NIC, DESY, Platanenallee 6, 15738 Zeuthen, Germany \\ Karl.Jansen@desy.de
}

Received 22 December 2009

Revised 14 April 2010

Communicated by T. Mendes

\begin{abstract}
We present lattice QCD simulation results from the European Twisted Mass Collaboration (ETMC). In particular, we present the non-perturbatively computed strange baryon spectrum, list a number of precisely determined low energy constants of chiral perturbation theory, and provide a first account of simulations including the strange and charm quark degrees of freedom.
\end{abstract}

Keywords: Lattice QCD; LEC; meson physics.

\section{Introduction}

In nature, we observe three generations of neutrinos as demonstrated by LEP. Correspondingly, there should exist three generations of quarks, ordered in quark pairs as (up, down), (strange, charm) and (bottom, top). The masses of these quarks show a particular hierarchy with essentially mass-degenerate up and down quarks and basically exponentially increasing mass values from the strange to the top quark.

We strongly believe nowadays that the quarks are the constituents of all hadrons with the gluons being the interaction particles that — via the strong interaction "glue" the quarks together to form the bound hadron states which are the ones observed in experiments. The mechanism of forming the bound states is theoretically described by quantum chromodynamics. The postulation of QCD is that at very short distances the quarks behave as almost free particles that interact only very weakly, a phenomenon we call asymptotic freedom. At large distances, at the order of $1 \mathrm{fm}$, the quarks interact extremely strongly, and in fact so strongly that they will never be seen as final, observable states but rather form the observed hadron bound spectrum. The latter phenomenon is called confinement of quarks.

Since the interaction between quarks becomes so strong at large distances, analytical methods such as perturbation theory fail to analyze QCD. A method to nevertheless tackle the problem is to formulate QCD on a four-dimensional, Euclidean space-time grid. This setup first of all allows for a rigorous definition of QCD and 
leads to fundamental theoretical and conceptual investigations. On the other hand, the lattice approach enables theorists to perform large scale numerical simulations. In this contribution, we will describe one approach to "lattice QCD", the twisted mass formulation.

In the past, lattice physicists had to work with a number of limitations when performing numerical simulations. These simulations are extremely expensive, reaching the need for Peta-flop computing and even beyond, a regime of computing power we just reach today. Therefore, for a long time the sea quarks were treated as infinitely heavy, indeed a crude approximation given that the up and down quarks have masses of only $\mathrm{O}(\mathrm{MeV})$. In this setup, the quarks were then treated only as valence quarks. In a next step, only the lightest quark doublet, the up and down quarks, were taken into consideration as sea quarks, although their mass values as used in the simulation had been unphysically large.

Nowadays, besides the up and down quarks, the strange quark is also included in the simulations. In addition, these simulations are performed in almost physical conditions, having the quarks masses close to their physical values, large lattices with about $3 \mathrm{fm}$ linear extent and small values of the lattice spacing such that a controlled continuum limit can be performed. With a box-size with linear extent of about $3 \mathrm{fm}$, a particle such as a proton with a radius of about $1 \mathrm{fm}$ fits well into the lattice and finite size effects are suppressed. The situation of the change of the simulation landscape is illustrated in Fig. 1(a). In the figure, the dot at $a=0 \mathrm{fm}$ indicates the physical point. The cross represents a state of the art simulation in the year 2001. As can be seen in the graph, most of the simulations now go well-beyond what could be reached in 2001, demonstrating clearly the progress in performing realistic simulations.

The drastic change in the situation is due to three main developments: (i) algorithmic breakthroughs; (ii) machine development (the computing power of the present BG/P systems is even outperforming Moore's law); (iii) conceptual developments, such as the use of improved actions which reduce lattice artefacts and the development of non-perturbative renormalization.

As a physical example of results we can achieve presently, we show in Fig. 1(b) the continuum extrapolated strange baryon spectrum as obtained ${ }^{1}$ by the European Twisted Mass Collaboration (ETMC), of which the author is a member. ETMC comprises 16 institutions in Europe, i.e., Cyprus (University of Cyprus), France (University of Paris Sud and LPSC Grenoble), Germany (Humboldt University zu Berlin, University of Münster, DESY in Hamburg and Zeuthen), Great Britain (University of Glasgow and University of Liverpool), Italy (University of Rome I, II and III, ECT* Trento), Netherlands (University of Groningen), Poland (University of Poznan), Spain (University of Valencia), Switzerland (University of Bern).

The baryon spectrum calculation has been considered a benchmark study for lattice QCD for a long time. It is therefore very reassuring that finally this important result can be obtained precisely from ab initio and non-perturbative lattice simulations. 

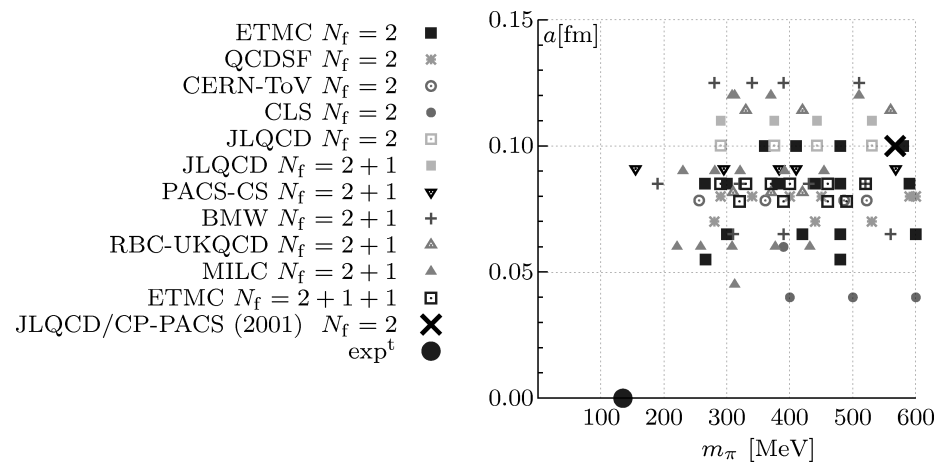

(a)

Baryon Spectrum

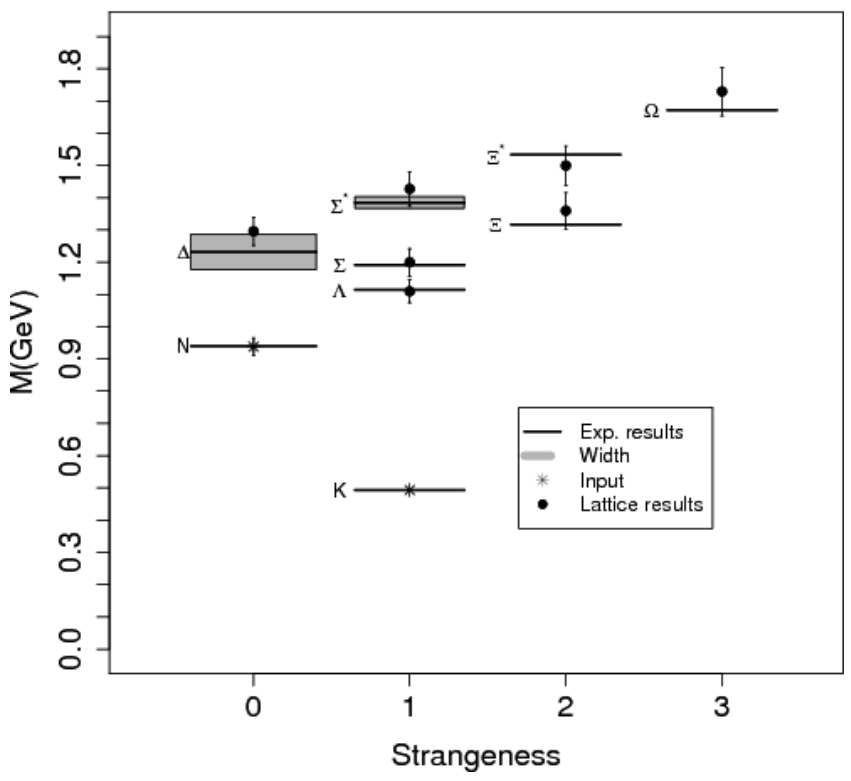

(b)

Fig. 1. (a) The values of the lattice spacing $a$ and pseudo-scalar masses $m_{\mathrm{PS}}$ as employed presently in typical QCD simulations by various collaborations as (incompletely) listed in the legend. The dot at $a=0$ indicates the physical point where in the continuum the pseudo-scalar meson assumes its experimentally measured value. The cross represents a state of the art simulation by the JLQCD collaboration in 2001. (b) The continuum strange baryon spectrum from the ETM collaboration.

\section{Twisted Mass Fermions}

Twisted mass fermions ${ }^{3,4}$ belong to the class of Wilson fermions. ${ }^{2}$ In this approach the lattice artefacts in physical observables appear only quadratically in the lattice spacing. This is in contrast to a standard Wilson action, where these lattice 
spacing effects are linear. The main advantage of the twisted mass formulation of lattice QCD is then that this kind of fermions provide an improved, i.e., $\mathcal{O}\left(a^{2}\right)$, continuum limit scaling of physical observables. The twisted mass formulation of Lattice $\mathrm{QCD}^{3,4}$ is being studied extensively with $N_{f}=2$ dynamical flavors, i.e., including only the lightest up and down quark doublet, by the European Twisted Mass collaboration see Ref. 8 and references therein.

The fermionic action for two flavors of twisted, mass-degenerate quarks in the so called twisted basis ${ }^{3,9}$ reads

$$
S_{\mathrm{tm}}=a^{4} \sum_{x}\left\{\bar{\chi}(x)\left[D[U]+m_{0}+i \mu_{q} \gamma_{5} \tau^{3}\right] \chi(x)\right\}
$$

where $m_{0}$ is the untwisted bare quark mass, $\mu_{q}$ is the bare twisted quark mass, $\tau^{3}$ is the third Pauli matrix acting in flavor space and

$$
D[U]=\frac{1}{2}\left[\gamma_{\mu}\left(\nabla_{\mu}+\nabla_{\mu}^{*}\right)-a \nabla_{\mu}^{*} \nabla_{\mu}\right]
$$

is the massless Wilson-Dirac operator. $\nabla_{\mu}$ and $\nabla_{\mu}^{*}$ are the forward and backward gauge covariant difference operators, respectively. Twisted mass fermions are said to be at maximal twist if the bare untwisted quark mass $m_{0}$ is tuned to its critical value $m_{\text {crit }}$, the situation we shall be interested in. For convenience we define the hopping parameter $\kappa=1 /\left(8+2 a m_{0}\right)$.

Maximally twisted mass fermions provide important advantages: the spectrum of $Q^{\dagger} Q$ with $Q=\gamma_{5}\left(D[U]+m_{0}+i \mu_{q} \gamma_{5}\right)$ is bounded from below, which was the original reason to consider twisted mass fermions. ${ }^{3}$ At maximal twist, the twisted quark mass $\mu_{q}$ is related directly to the physical quark mass and renormalizes multiplicatively only. Many mixings under renormalization are expected to be simplified. ${ }^{4,9}$ Also, physical observables are automatically $\mathcal{O}(a)$ improved. Another feature of maximally twisted mass fermions is that the pseudo-scalar decay constant $f_{\mathrm{PS}}$ does not need any renormalization which allows for a very precise determination of this quantity.

The main drawback of maximally twisted mass fermions is that both parity and flavor symmetry are broken explicitly at nonzero values of the lattice spacing. However, it turns out that this is presumably only relevant for the mass of the neutral pseudo-scalar meson (and kinematically related quantities). ${ }^{10}$

\section{Results for Two Flavors of Mass-Degenerate Quarks}

Since in the maximal twist situation the theory is $\mathcal{O}(a)$-improved leading lattice artefacts are expected to be of order $a^{2}$. This can be checked by extrapolating a physical quantity in units of the force parameter ${ }^{11} r_{0}^{\chi}$ extrapolated to the chiral limit at fixed physical situation to the continuum limit. We show two such examples in Fig. 2. In the left panel we show $r_{0}^{\chi} f_{\mathrm{PS}}$ as a function of $\left(a / r_{0}^{\chi}\right)^{2}$ at fixed value of $r_{0}^{\chi} m_{\mathrm{PS}}$. In order to match the values of $r_{0}^{\chi} m_{\mathrm{PS}}$ at each value of $r_{0}^{\chi} / a$ and to fix the volume to $r_{0}^{\chi} \cdot L=5$ we had to perform short interpolations or extrapolations. The 


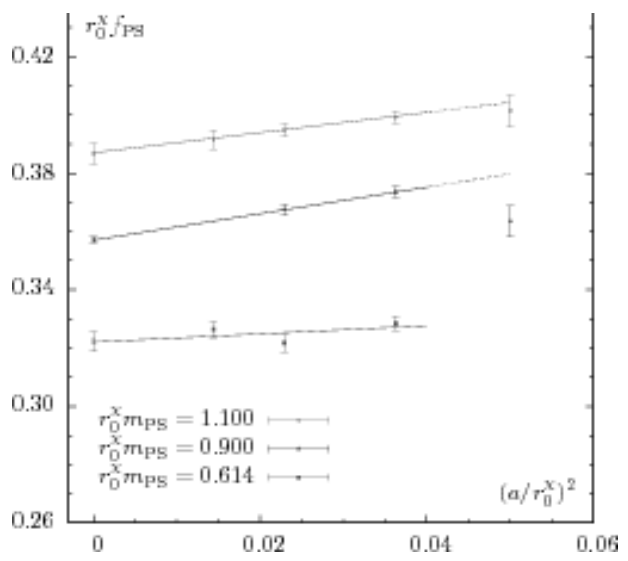

(a)

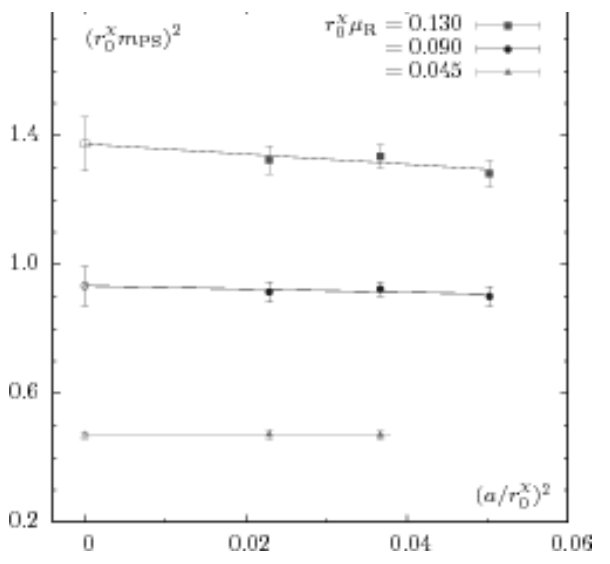

(b)

Fig. 2. Scaling in finite, fixed volume for $r_{0}^{\chi} f_{\mathrm{PS}}$ at fixed values of $r_{0}^{\chi} m_{\mathrm{PS}}(\mathrm{a})$ and for $\left(r_{0}^{\chi} m_{\mathrm{PS}}\right)^{2}$ at fixed values of $r_{0}^{\chi} \mu_{R}$ (b). The data are plot with decreasing values of both quantities from top to bottom. In (b) we cannot include data at $\beta=4.2$ due to the missing value of the renormalization factor of the pseudo-scalar current $Z_{\mathrm{P}}$.

straight lines are linear fits in $\left(a / r_{0}^{\chi}\right)^{2}$ to the corresponding data, with the data at the largest value of the lattice spacing not being included in the fit. It is clearly visible that the lattice artefacts appear to scale linearly in $a^{2}$ and that their overall size is small.

In the right panel of Fig. 2 we show the scaling of $r_{0}^{\chi} m_{\mathrm{PS}}$ as a function of $\left(a / r_{0}^{\chi}\right)^{2}$ at fixed values of the renormalized quark mass $r_{0}^{\chi} \mu_{R}$, again at fixed, finite volume. We conclude that also the charged pseudo-scalar mass has only small lattice artefacts.

The dependence of $m_{\mathrm{PS}}$ and $f_{\mathrm{PS}}$ on the renormalized quark mass and volume can be described by chiral perturbation theory $(\chi \mathrm{PT}) \cdot{ }^{13,14}$ The residual lattice artefacts of order $a^{2}$ can also be included in the analysis. The corresponding formulae can be found in Refs. 8 and 15. We fit these formulae to our data in order to extract the parameters of the $N_{f}=2$ chiral Lagrangian, i.e., the low energy constants and some derived quantities. Moreover, we can use these fits to calibrate our lattices by determining the value of the renormalized quark mass $r_{0}^{\chi} \mu_{R}$ where the ratio $m_{\mathrm{PS}} / f_{\mathrm{PS}}$ assumes its physical value (i.e., $m_{\pi} / f_{\pi}$ ), and set $f_{\mathrm{PS}}=f_{\pi}=130.7 \mathrm{MeV}$ there, as done in Ref. 5 . Hence, $f_{\pi}$ is used in this paper to set the scale.

The results of these fits can be found in Table 1 . We give statistical and systematic errors separately, the systematic one being asymmetrical. The results are obtained by performing $\mathcal{O}(80)$ fits, which differ in fit-range, finite size correction formulae and in the order of $\chi \mathrm{PT}$. The final result is obtained as the median of the corresponding weighted distribution over all fits. The statistical error is determined using the bootstrap method with 1000 samples. The systematic uncertainty is estimated from the $68 \%$ confidence interval of the weighted distribution. For details 
Table 1. Summary of fit results, determined from the weighted distribution of a number of $\mathcal{O}(80)$ different fits. The first error is of statistical origin while the second, the asymmetric one, accounts for the systematic uncertainties. $\Sigma$ and $m_{u, d}$ are renormalized in the $\overline{\mathrm{MS}}$ scheme at the renormalization scale $\mu=2 \mathrm{GeV}$, as the values of $Z_{\mathrm{P}}$ are in the $\overline{\mathrm{MS}}$ scheme at scale $2 \mathrm{GeV}$. The scale is set by $f_{\pi}=130.7 \mathrm{MeV}$.

\begin{tabular}{lccc}
\hline & Median & Statistical & Systematic \\
\hline$m_{\text {up }, \text { down }}(\mathrm{MeV})$ & 3.54 & $(19)$ & $(+16-17)$ \\
$\bar{\ell}_{3}$ & 3.50 & $(9)$ & $(+9-30)$ \\
$\bar{\ell}_{4}$ & 4.66 & $(4)$ & $(+4-33)$ \\
$f_{0}(\mathrm{MeV})$ & 121.5 & $(0.1)$ & $(+1.1-0.1)$ \\
$r_{0}(\mathrm{fm})$ & 0.420 & $(9)$ & $(+10-11)$ \\
$|\Sigma|^{1 / 3}(\mathrm{MeV})$ & 270 & $(5)$ & $(+3-4)$ \\
$f_{\pi} / f_{0}$ & 1.0755 & $(6)$ & $(+8-94)$ \\
\hline
\end{tabular}

see Ref. 8. The fit results for the determination of physical quantities and the low energy constants listed in Table 1 belong to the most precise determination of their kind worldwide.

\section{Results Adding Dynamical Strange and Charm Quarks}

The very nice results for mass-degenerate quarks discussed in the last section motivate to go one step beyond this setup. The ETM collaboration has by now included the strange and the charm degrees of freedom in their simulations and they are the first collaboration to perform such studies.

The fermionic action for the light doublet is given by the same form as given in Eq. (1). In the heavy sector, the action becomes:

$$
S_{h}=a^{4} \sum_{x}\left\{\bar{\chi}_{h}(x)\left[D[U]+m_{0, h}+i \mu_{\sigma} \gamma_{5} \tau_{1}+\mu_{\delta} \tau_{3}\right] \chi_{h}(x)\right\} .
$$

The heavy doublet mass parameters $\mu_{\sigma}$ and $\mu_{\delta}$ should be adjusted in order to reproduce the values of the renormalized strange $(s)$ and charm $(c)$ quark masses. The latter are related to $\mu_{\sigma}$ and $\mu_{\delta}$ via Ref. 18:

$$
\left(m_{s, c}\right)_{R}=\frac{1}{Z_{P}}\left(\mu_{\sigma} \mp \frac{Z_{P}}{Z_{S}} \mu_{\delta}\right),
$$

where the minus sign corresponds to the strange and the plus sign to the charm quark. In practice we fix the values $\mu_{\sigma}$ and $\mu_{\delta}$ by requiring the resulting $K$ - and $D$-meson masses to match experimental results.

Tuning to maximal twist requires to set $m_{0, l}$ and $m_{0, h}$ to a sufficiently accurate value of the critical mass $m_{\text {crit }}=m_{\text {crit }}(\beta) .{ }^{18}$ Here we set $m_{0, l}=m_{0, h} \equiv$ $1 /(2 \kappa)-4$. As shown in Refs. 18 and 19, this is consistent with $\mathcal{O}(a)$ improvement defined by the maximal twist condition $a m_{\mathrm{PCAC}, l}=0$. We demand that the ratio $\left|Z_{\mathrm{A}} a m_{\mathrm{PCAC}} / a \mu_{q}\right| \lesssim 0.1$ and also that its error $\Delta\left(Z_{\mathrm{A}} a m_{\mathrm{PCAC}} / a \mu_{q}\right) \lesssim 0.1$. These criteria and their justification for tuning to maximal twist have been discussed in Ref. 7 . Note that we tune to $m_{\text {crit }}(\beta)$ at each value of $\mu_{q}$ separately. 

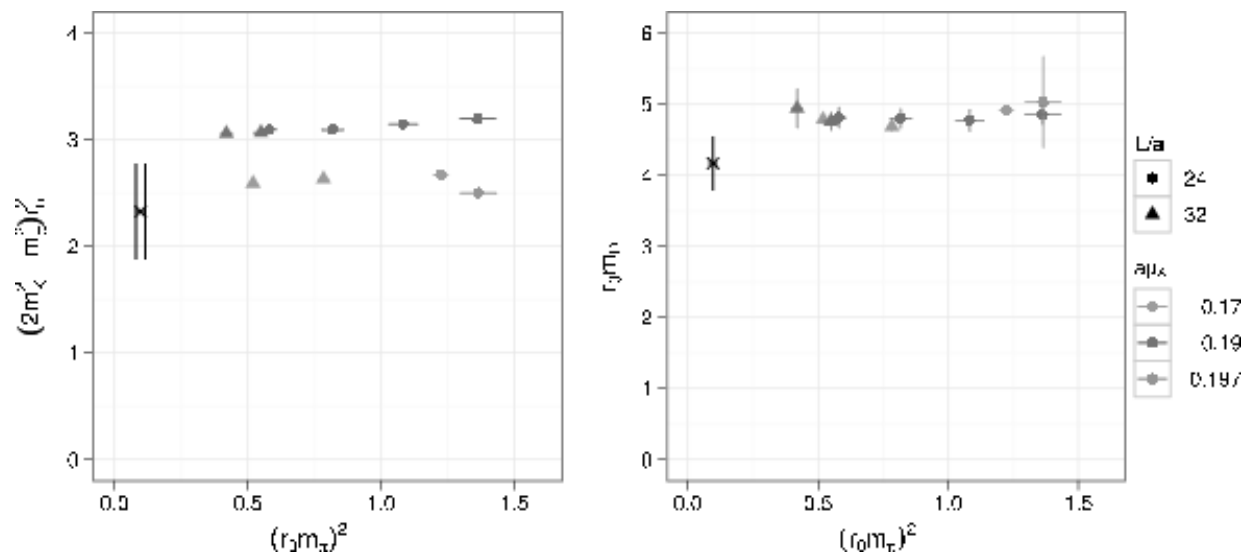

Fig. 3. $r_{0}^{2}\left(2 m_{K}^{2}-m_{\pi}^{2}\right)$ and $r_{0} m_{D}$ as functions of $\left(r_{0} m_{\pi}\right)^{2}$, showing the status of the tuning of the strange and charm quark mass respectively. The experimental value from PDG is added as the cross $\left(r_{0}=0.44(4)\right.$ fm was used). We show points at $\beta=1.95$ and $\beta=1.90$ runs (upper lying points in the left panel). The most right, low-lying point in the left panel corresponds to $\beta=1.90$ with a different heavy sector splitting $a \mu_{\delta}$. Circles denote runs with $L / a=24$, triangles indicate a volume with $L / a=32$.

A most interesting result in this $N_{f}=2+1+1$ setup is the calculation of the $K$ - and $D$-meson sector. Leaving out technical details of this complicated and demanding calculation, we show in the left panel of Fig. 3 the tuning of the strange quark mass by showing the difference, scaled with the chirally extrapolated value of $r_{0} / a$ between twice the $K$-meson mass squared and the pion mass squared. For our coarse value of the lattice spacing at $\beta=1.90$ the data appear to overshoot the physical point (the cross on the left), while at our finer value of the lattice spacing at $\beta=1.95$ the $K$-meson mass extrapolates better. To improve the tuning of the strange quark mass, we are currently applying a reweighting procedure as described in Ref. 20 in the parameters $a \mu_{\delta}$ and $\kappa$.

In the right panel of Fig. 3 the mass of the $D$-meson as a function of the pion mass squared is shown for various simulation points as well as the experimental value from the Particle Data Group. ${ }^{16}$ The plot demonstrates that we have tuned the charm (sea) quark mass in our simulations to a physically realistic value. As a final check, we also use an estimate of $Z_{P} / Z_{S}$ to verify that $m_{c} \sim 10 m_{s}$.

In the light meson sector we can perform a similar analysis as discussed above for the case of two mass-degenerate flavors of quarks. Assuming that $Z_{P}$ is effectively a function of $\beta$ in the range of parameters we are considering, we can fit the ratio of those $Z_{P}$-values and lattice spacings and extract lattice spacings from the combined fit. In every fit we use as inputs the physical $f_{\pi}$ and $m_{\pi}$, and extract $f_{0}, \bar{l}_{3}$ and $\bar{l}_{4}$.

Using $f_{\pi}=130.7 \mathrm{MeV}$ as our physical input parameter, we find from the fit a value of the lattice spacing of $a \approx 0.086 \mathrm{fm}$ at $\beta=1.9$ and $a \approx 0.078 \mathrm{fm}$ at $\beta=1.95$. Comparing the results of Table 2 with those of Table 1 , we find a very good agreement between our $N_{f}=2$ and $N_{f}=2+1+1$ simulations. This fact and the comparison of $f_{\mathrm{PS}}$ and $m_{\mathrm{PS}}$ directly as done in Ref. 17 makes us confident that 
Table 2. Results from the NLO $S U(2) \chi \mathrm{PT}$ fits for combined, for data only at $\beta=1.9$ and only at $\beta=1.95$, respectively.

\begin{tabular}{lccc}
\hline$\beta$ & $f_{0}(\mathrm{MeV})$ & $\bar{l}_{3}$ & $\bar{l}_{4}$ \\
\hline $1.9,1.95$ & $121(4)$ & $3.5(2)$ & $4.7(2)$ \\
1.9 & $121(4)$ & $3.4(2)$ & $4.8(2)$ \\
1.95 & $121(4)$ & $3.7(2)$ & $4.7(2)$ \\
\hline
\end{tabular}

also for the situation of $N_{f}=2+1+1$ flavors of quarks the lattice spacing scaling violations will be small.

\section{Conclusion}

In this contribution we have discussed a particular formulation of lattice QCD, maximally twisted mass fermions. We have shown that with this $\mathcal{O}(a)$-improved action, precise results in the light meson and the strange baryon sector can be obtained. In the course of this investigation, it has been demonstrated that twisted maximal twisted mass fermions indeed scale with $a^{2}$ towards the continuum limit and that even these remaining $a^{2}$ corrections are very small giving rise to a wellcontrolled continuum limit extrapolation of lattice results. Fitting the data for $f_{\pi}$ and $m_{\pi}$ it became possible to extract a number of physical quantities and low energy constants of chiral perturbation theory very precisely see Table 1 .

Encouraged by these results, we have described first simulation results for the situation when a dynamical strange and charm quark are included. We have demonstrated that it is possible to tune to physical $K$ - and $D$-meson masses. In addition, the good agreement of the $N_{f}=2$ and $N_{f}=2+1+1$ simulations, visible when comparing Tables 1 and 2, indicates that also for the latter case lattice artefacts, at least in the light quark sector, are small. This offers the promising possibility to obtain precise physical results also with dynamical up, down, strange and charm simulations for the first time.

\section{Acknowledgments}

We thank all other members of the ETM Collaboration for a very fruitful collaboration and many valuable discussions. The HPC resources for this project have been made available by the computer centers of Barcelona, Groningen, Jülich, Lyon, Munich, Paris and Rome (apeNEXT), which we thank for enabling us to perform this work. This work has also been supported in part by the DFG Sonderforschungsbereich/Transregio SFB/TR9-03, and by GENCI (IDRIS-CINE S), Grant 2009-052271.

\section{References}

1. C. Alexandrou et al., [arXiv:0910.2419 hep-lat].

2. K. G. Wilson, Phys. Rev. D 10 (1974) 2445.

3. ALPHA Collaboration, R. Frezzotti et al., J. High Energy Phys. 08 (2001) 058. 
4. R. Frezzotti and G. C. Rossi, J. High Energy Phys. 10 (2004) 070.

5. ETM Collaboration, P. Boucaud et al., Phys. Lett. B 650 (2007) 304.

6. ETM Collaboration, B. Blossier et al., J. High Energy Phys. 04 (2008) 020.

7. ETM Collaboration, P. Boucaud et al., Comput. Phys. Commun. 179 (2008) 695.

8. ETM Collaboration, R. Baron et al. [arXiv:0911.5061 hep-lat].

9. R. Frezzotti and G. C. Rossi, J. High Energy Phys. 08 (2004) 007.

10. ETM Collaboration, P. Dimopoulos et al. [arXiv:0908.0451 hep-lat].

11. R. Sommer, Nucl. Phys. B 411 (1994) 839.

12. S. Weinberg, Physica A 96 (1979) 327.

13. J. Gasser and H. Leutwyler, Ann. Phys. 158 (1984) 142.

14. J. Gasser and H. Leutwyler, Nucl. Phys. B 250 (1985) 465.

15. G. Colangelo, S. Dürr and C. Haefeli, Nucl. Phys. B 721 (2005) 136.

16. Particle Data Group Collaboration, C. Amsler et al., Phys. Lett. B 667 (2008) 1.

17. ETM Collaboration, R. Baron et al. [arXiv:0911.5244 hep-lat].

18. R. Frezzotti and G. C. Rossi, Nucl. Phys. Proc. Suppl. 128 (2004) 193.

19. T. Chiarappa et al., Eur. Phys. J. C 50 (2007) 373.

20. ETM Collaboration, R. Baron et al., Proceedings of Science LAT2008 (2008) 094. 